\title{
Triple gastric ulcer in young child - case presentation
}

\author{
Smaranda DIACONESCU ${ }^{1}$, Marin BURLEA ${ }^{1}$, Raluca STANCA ${ }^{2}$, Monica ALEXOAE ${ }^{1}$, lleana IONIUC ${ }^{1}$ \\ ${ }^{1}$ "Gr. T. Popa" University of Medicine and Pharmacy, lasi, Romania \\ ${ }^{2}$ „Sf. Maria“ Emergency Hospital for Children, lasi, Romania
}

\begin{abstract}
Peptic ulcer is represented by loss of gastric or duodenal mucosal substance. It's been considered a rare disease at the paediatric age. The main cause is represented by Helicobacter pylori infections or drugs administration. The authors present a case of nine-year-old child with triple gastric ulcer without Helicobacter pylori infection or prior treatment with nonsteroidal anti-inflammatory drugs or other medication with possible impact.
\end{abstract}

Keywords: child, ulcer, Helicobacter pylori infection

\section{INTRODUCTION}

Peptic ulcer is represented by loss of gastric or duodenal mucosal substance with submucosal exposure. The aetiology of this disease is multifactorial involving the imbalance between the aggressive and defeating factors, the hereditary and psychological factors. It's been considered a rare disease at the pediatric ages, but its incidence is ranging between $8.1 \%$ in Europe and $17.4 \%$ in United States. The most frequent cause of peptic ulcer in paediatrics is represented by Helicobacter pylori infection and rarely by the administration of nonsteroidal anti-inflammatory drugs (NSAIDs), corticoids and other immunosupressives, as well as psychological stress. (1)

\section{CASE REPORT}

A nine-year-old boy was admitted to our paediatric gastroenterology unit for abdominal pain, vomiting, loss of appetite and melena. Medical history revealed frequents respiratory infections treated with antibiotics and symptomatic drugs, including administration of antipyretics until the age of six. Family history showed that both his parents and maternal grandmother had Helicobacter pylori infection and maternal grandfather had perforated gastric ulcer surgically treated. The boy presented mild diffuse abdominal pain started from approximately one month prior to admission. Two days before presenting to the hospital, once with a psychological stress (sport competition), the pain became important and the boy started to vomit (alimentary and bilious) and presented melena. Physical examination at admission revealed an a well-developed and well-nourished boy, with grade I obesity $(\mathrm{W}=50 \mathrm{~kg}, \mathrm{H}=130 \mathrm{~cm}, \mathrm{BMI}-30$ $\left.\mathrm{kg} / \mathrm{m}^{2}\right)$; vital signs: temperature $98.4 \mathrm{~F}$, pulse 87 bpm, respiratory rate $18 / \mathrm{m}$, blood pressure $115 / 78 \mathrm{mmHg}$; normal shaped head, no sign of cranial trauma, equal and reactive pupils, normal nostrils, was well hydrated mouth without oral lesions, moist mucous membranes, pale skin colour; normal pulmonary and cardiac auscultation; epigastric and umbilical pain without muscular defence, positive bowel sounds, mele- 
na, no hepatosplenomegaly was noted; warm extremities without clubbing, oedema or cyanosis; no neurologic focal deficits. Laboratory data was within normal limits including serum gastrinemia. The child underwent an upper digestive endoscopy which revealed diffuse congestion in the lower oesophagus, normal cardia, hyperaemic gastric mucosa with a $2 \mathrm{~cm}$ linear ulcer Forrest III with oedematous base and fibrin and another two linear ulcers of $0.5-1 \mathrm{~cm}$ in the antral region. (Figure 1)

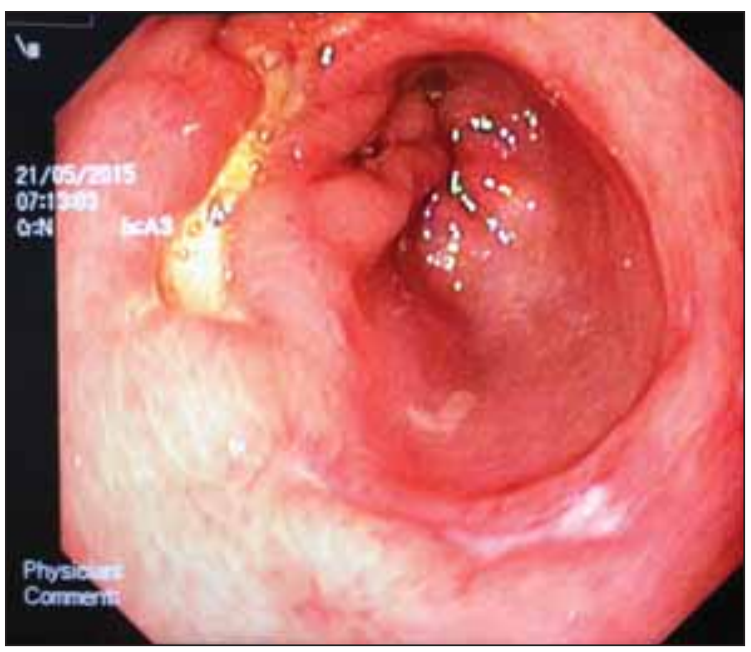

FIGURE 1. Three linear ulcers in the antrum (endoscopic image)

Rapid urease test and histology were negative for Helicobacter pylori. Proton-pump inhibitors (PPIs) were started; the patient was released from hospital after three days with diet recommendation and PPIs recommendation (20 $\mathrm{mg} /$ day) for two months. The two month checkup showed an asymptomatic child and the control endoscopy visualized a normal gastric mucosa with a single linear scar at the gastric antrum (Figure 2).

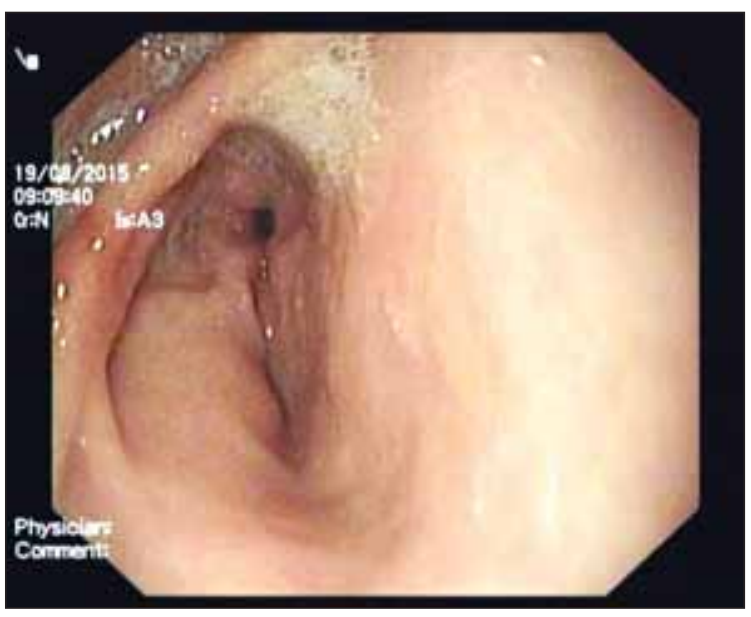

FIGURE 2. Normal gastric mucosa; linear scar at the antrum (endoscopic image at 2 months follow-up)
Helicobacter pylori testing was likewise previously negative. Except dietary prescriptions no medication was further recommended.

\section{DISCUSSIONS}

Peptic ulcer is rarely suspected in children with abdominal pain. The most frequent symptoms are mild epigastric or diffuse abdominal pain, which disappear immediately after ingestion of food or antacids, abdominal meteorism, heartburns, eructation, nausea, vomiting, weight loss. Gastrointestinal haemorrhage (hematemesis or melena) is the leading complication, together with perforation and, rarely, pyloric stenosis. The upper digestive endoscopy is the gold standard in diagnostic. The investigation can be performed at any age and allows a direct view of the esophagus, stomach and duodenum, showing the specific lesions. In addition, it allows sampling biopsies for histological examination and for Helicobacter pylori infections diagnosis by rapid urease test. Therapeutic manoeuvers of endoscopic haemostasis scan also be performed: clipping, injections or argon plasma coagulation. (2) The treatment of peptic ulcer includes proton-pump inhibitors administered orally or intravenous (in patients with haemorrhage), Helicobacter pylori infection eradication using various associations of antibiotics according to international guidelines and surgical treatment in selected cases. In drug-induced ulcers (i.e.nonsteroidal anti-inflammatory drugsNSAIDs) the interruption of the administration is mandatory. (3)

In our case we didn't find any argument for the development of secondary ulcers, such recent consumption of NSAIDs or endogenous stress (caused by pathological condition as sepsis, chronic diseases, burns or trauma). Moreover we could not demonstrate the presence of Helicobacter pylori. Familial history can be perceived as a risk factor in this case, as well as the circumstances of occurrence of the hemorrhagic episode - psychological stress due to a sport competition. Even if family aggregation is obvious, however, only the existence of family history does not constitute a predictive factor for ulcers. Studies from the pre-H.pylori era suggested a polygenic inheritance in the familial aggregation of peptic ulcer as relatives of patients with gastric ulcer have a threefold increase in the prevalence of gastric but not of duodenal ulcer. (4) These evidences are supported by twin studies that provide evidence for a genetic predispo- 
sition to PUD that is independent of any predisposition to $\mathrm{H}$ pylori infection. (5) Psychological factors are difficult to quantify, but authors report a relationship between childhood physical abuse and peptic ulcers. (6) Other childhood adversities involved were long-lasting financial difficulties in the family, serious conflicts in the family including parental divorce or family member seriously or chronically ill. (7) Some studies reported cases of peptic ulcers apparently occurring without any risk factor. (8) If a leading cause is detected the prognostic is very good, the complication rate being 1-2\% per year in general population. (9) It has been shown that the prophylactic treatment with protonpump inhibitors considerably decreased the risk of gastric or duodenal ulcers relapses. (10) Prolonged treatment for up to one year with antisecretory medications is indicated in patients with increased risk (recurrent complicated or giant ulcers). (10) The improvement of quality and ac- curacy of diagnosis tests, as well as the detailed physical examination, determines a low rate of complication of these diseases. The treatment strategy is multimodal, including endoscopic haemostatic manoeuvres in pediatric patients. In our case there is an unusual association between endoscopic findings (multiple gastric lesions) with severe symptomatology (melena) with surprising causative factors as familial aggregation and psychological stress.

\section{CONCLUSIONS}

Even the incidence of non Helicobacter pylori ulcers is low in pediatric population, in children with suggestive symptoms and a positive family history, it is necessary to perform an esophago-gastroduodenoscopy that could reveal underlying lesions with a crucial importance in therapeutic strategies.

\section{REFERENCES}

1. Guariso G., Gasparetto M. - Update on Peptic Ulcers in the Pediatric Age, Ulcers, 2012; Article ID 896509 doi:10.1155/2012/ 896509

2. Kliegman R.M., Stanton B.F., St. Geme J.W., Schor N.F. - Nelson Textbook of Pediatrics, 19th edition, 2011, pg 1291-1294.

3. Lee N.M., Yun S.W., Chae S.A., Yoo B.H., Cha S.J., Kwak B.K. - Perforated duodenal ulcer presenting with massive hematochezia in a 30-month-old child. World Journal of Gastroenterology: WJG. 2009; 15(38):4853-4855. doi:10.3748/ wjg.15.4853.

4. Rotter J.I. - Peptic ulcer. In: The principles and practice of medical genetics, Emery A.E.H., Rimoin D.L. (Eds), Churchill Livingstone, New York 1983. p.863.
5. Malaty H.M., Graham D.Y., Isaksson I. et al. - Are genetic influences on peptic ulcer dependent or independent of genetic influences for Helicobacter pylori infection? Arch Intern Med 2000; 160:105.

6. Fuller-Thomson E., Bottoms J., Brennenstuhl S., Hurd M. - Is childhood physical abuse associated with peptic ulcer disease? Findings from a population-based study. J Interpers Violence. 2011; 26(16):3225-47. doi: 10.1177/08862605103 93007

7. Sumanen M.P., Koskenvuo M.J., Sillanmäki L.H., Mattila K.J. - Peptic ulcer and childhood adversities experienced by working-aged people. World J Gastroenterol. 2009; 15(27):3405-10.

8. Sýkora J., Rowland M. - Helicobacter pylori in pediatrics, Helicobacter, 2011; 16(1):59-64
9. Brown K., Lundborg P., Levinson P., et al. - Incidence of peptic ulcer bleeding in the US pediatric population, Journal of Pediatric Gastroenterology and Nutrition, 2012; 54(6):733-736

10. Lai K.C., Lam S.K., Chu K.M et al. Lansoprazole reduces ulcer relapse after eradication of Helicobacter pylori in nonsteroidal anti-inflammatory drug users - a randomized trial, Alimentary Pharmacology and Therapeutics, 2003; 18(8):829-836

11. Soplepmann J., Peetsalu M., Toomas V., Peetsalu A. - Giant duodenal posterior wall ulcers complicated with hemorrhage: Long-term results of surgical treatment, Lithuanian Surgery, 2004; 2(4):286-292 